\title{
Application of the Two Nonzero Component Lemma in Resource Allocation
}

\author{
Morteza Seddighin \\ Mathematics Department, Indiana University East, Richmond, IN, USA \\ Email: mseddigh@indiana.edu \\ Received 16 April 2014; revised 16 May 2014; accepted 21 May 2014 \\ Copyright (C 2014 by author and Scientific Research Publishing Inc. \\ This work is licensed under the Creative Commons Attribution International License (CC BY). \\ http://creativecommons.org/licenses/by/4.0/

\begin{abstract}
In this paper we will generalize the author's two nonzero component lemma to general self-reducing functions and utilize it to find closed from answers for some resource allocation problems.
\end{abstract}

\section{Keywords}

Two Nonzero Component Lemma, Resource Allocation, The Distribution of the Search Effort

\section{Introduction}

The technique we will use in this paper was first applied by this author to problems in matrix inequalities and matrix optimization. Historically, many researchers have established matrix inequalities by variational methods. In a variational approach one differentiates the functional involved to arrive at an "Euler equation" and then solves the Euler Equation to obtain the minimizing or maximizing vectors of the functional. The same technique is also often used in matrix optimization. Solving the Euler equations obtained are tedious and generally provide little information. Others have established inequalities for matrices and operators by going through a two-step process which consists of first computing upper bounds for suitable functions on intervals containing the spectrum of the matrix or operator and then applying the standard operational calculus to that matrix. This method, which we refer to as "the operational calculus method", has the following two limitations: First,it does not provide any information about vectors for which the established inequalities become equalities (a matrix optimization problem). Second, the operational calculus method is futile in extending Kantorovich-type inequalities to operators on infinite dimensional Hilbert spaces. See [1] for examples using each of the two methods mentioned above. In his study of matrix inequalities and matrix optimization, the author has discovered and proved a lemma called the Two Nonzero Component Lemma or TNCL for short. In this paper we will state an extension of the author's Two Nonzero Component Lemma and utilize it to solve some resource allocatoin problems. While resource allocatoin problems are not generally formulated in terms of matrices, as we will see, 
there are some similarities between matrix optimization problems and resource allocation problems Let us first state the TNCL as it was used in author's previous papers on matrix inequalities and matrix optimization.

\section{The Two Nonzero Component Lemma}

It was in his investigation on problems of antieigenvalue theory that the author discovered and proved the Two Nonzero Component Lemma (see [2]-[4]). Although this lemma is utilized effectively by the author in matrix theory, it is by nature a dimension reducing optimization lemma which has potential applications in all areas of mathematics and physics. While TNCL was implicitly used in all of the papers just cited, it was not until 2009 that the author stated a formal description of the lemma in his paper titled, "Antieigenvalue Techniques in Statistics" (see [4]). Below is the statement of the lemma. For the proof of the lemma please see the author's work cited above.

Lemma 1 (The Two Nonzero Component Lemma) Let $l_{1}^{+}$be the set of all sequences with nonnegative terms in the Banach Space $l_{1}$. That is, let

$$
l_{1}^{+}=\left\{\mathbf{t}=\left(t_{i}\right) \varepsilon l_{1}, t_{i} \geq 0\right\} .
$$

Let

$$
F\left(x_{1}, x_{2}, \cdots x_{m}\right)
$$

be a function from $R^{m}$ to $R$. Assume $g_{k}(\mathbf{t})=\sum c_{i}^{k} t_{i}$ for $\left(c_{i}^{k}\right) \in l_{1}^{+}, \mathbf{t} \in l_{1}^{+}$, and $1 \leq k \leq m$. Then the minimizing vectors for the function

$$
F\left(g_{1}(\mathbf{t}), g_{2}(\mathbf{t}), \cdots g_{m}(\mathbf{t})\right)
$$

on the convex set $C=\left\{\left(t_{i}\right) \in l_{1}: \sum t_{i}=1\right\}$ have at most two nonzero components.

What make the lemma possible are the following two facts: First, the fact that the set

$$
C=\left\{\left(t_{i}\right) \in l_{1}^{+}: \sum t_{i}=1\right\}
$$

is convex. Second, a special property that the functions

$$
F\left(g_{1}(\mathbf{t}), g_{2}(\mathbf{t}), \cdots g_{m}(\mathbf{t})\right)
$$

involved possess. If we set

$$
D\left(t_{1}, t_{2}, t_{3}, \cdots\right)=F\left(g_{1}(\mathbf{t}), g_{2}(\mathbf{t}), g_{3}(\mathbf{t}), \cdots\right)
$$

then all restrictions

$$
D\left(t_{1}, t_{2}, \cdots, t_{i-1}, 0, t_{i+1}, \cdots\right)
$$

of

$$
D\left(t_{1}, t_{2}, t_{3}, \cdots\right)
$$

obtained by setting one component $t_{i}$ equal to zero, have the same algebraic form as $D\left(t_{1}, t_{2}, t_{3}, \cdots\right)$ itself. We call functions with this property self-reducing functions. Please note that TNCL is valid for both finite and infinite variable cases. Let us look at an example of a self-reducing function where there are only a finite number of variables $t_{1}, t_{2}, t_{3} \cdots t_{n}$ involved. Considered the function

$$
\frac{\beta_{1} t_{1}+\beta_{2} t_{2}+\cdots+\beta_{n} t_{n}}{\left|\lambda_{1}\right|^{2} t_{1}+\left|\lambda_{2}\right|^{2} t_{2}+\cdots+\left|\lambda_{3}\right|^{2} t_{3}}
$$

where $\beta_{1}, \beta_{2}, \beta_{3}, \cdots \beta_{n}$ are real numbers and $\lambda_{1}, \lambda_{2}, \cdots \lambda_{n}$ are complex numbers (this function appeared in [2]). Let

$$
D\left(t_{1}, t_{2}, \cdots, t_{n}\right)=\frac{\beta_{1} t_{1}+\beta_{2} t_{2}+\cdots+\beta_{n} t_{n}}{\left|\lambda_{1}\right|^{2} t_{1}+\left|\lambda_{2}\right|^{2} t_{2}+\cdots+\left|\lambda_{3}\right|^{2} t_{3}}
$$


then we have

$$
D\left(0, t_{2}, \cdots, t_{n}\right)=\frac{\beta_{2} t_{2}+\cdots+\beta_{n} t_{n}}{\left|\lambda_{2}\right|^{2} t_{2}+\cdots+\left|\lambda_{3}\right|^{2} t_{3}}
$$

which has the same algebraic form as

$$
D\left(t_{1}, t_{2}, \cdots, t_{n}\right)=\frac{\beta_{1} t_{1}+\beta_{2} t_{2}+\cdots+\beta_{n} t_{n}}{\left|\lambda_{1}\right|^{2} t_{1}+\left|\lambda_{2}\right|^{2} t_{2}+\cdots+\left|\lambda_{3}\right|^{2} t_{3}} .
$$

Indeed, for any $j, 1 \leq j<n$; all restrictions of the function

$$
D\left(t_{1}, t_{2}, \cdots, t_{n}\right)=\frac{\beta_{1} t_{1}+\beta_{2} t_{2}+\cdots+\beta_{n} t_{n}}{\left|\lambda_{1}\right|^{2} t_{1}+\left|\lambda_{2}\right|^{2} t_{2}+\cdots+\left|\lambda_{3}\right|^{2} t_{3}}
$$

obtained by setting an arbitrary set of $j$ components of $D\left(t_{1}, t_{2}, \cdots, t_{n}\right)$ equal to zeros have the same algebraic form as $D\left(t_{1}, t_{2}, \cdots, t_{n}\right)$.

Obviously, not all functions have this property. For instance, for the function $G\left(t_{1}, t_{2}\right)=2 t_{1}+t_{1} t_{2}$, we have $G\left(t_{1}, 0\right)=2 t_{1}$, which does not have the same algebraic form as $G\left(t_{1}, t_{2}\right)$. Note that functions $g_{i}(\mathbf{t})$ appearing in the statement of TNCL above are finite or infinite linear combinations of $t_{1}, t_{2}, t_{3}, \cdots$ The lemma was originally stated this way because when we deal with a matrix or operator optimization problem each $g_{i}(\mathbf{t})$ is either a finite or infinite linear combination of variables $t_{1}, t_{2}, t_{3}, \cdots$.

Example 2 In Theorem 1 of [4] we used TNCL to find the minimum of a Rayleigh quotient. A Rayleigh quotient of a positive operator $C$ over positive operators $A$ and $B$ is a quotient of the form

$$
\frac{(C f, f)}{(A f, f)^{\frac{1}{2}}(B f, f)^{\frac{1}{2}}}
$$

The unit vectors $f$ for which the minimum in

$$
\inf _{A f \neq 0, B f \neq 0} \frac{(C f, f)}{(A f, f)^{\frac{1}{2}}(B f, f)^{\frac{1}{2}}}
$$

is attained are called stationary values for (14). In Theorem 1 of [4] the minimum of (14) was found by converting the problem to the problem of finding the minimum of

$$
\begin{aligned}
& \frac{\left(\sum_{i=1}^{n} \lambda_{i} t_{i}\right)^{2}}{\left(\sum_{i=1}^{n} \alpha_{i} t_{i}\right)\left(\sum_{i=1}^{n} \beta_{i} t_{i}\right)} \\
& \text { Subject to } \sum_{i=1}^{n} t_{i}=1 .
\end{aligned}
$$

In this case $g_{1}(t)=\sum_{i=1}^{n} \lambda_{i} t_{i}, g_{2}(t)=\sum_{i=1}^{n} \alpha_{i} t_{i}$, and $g_{3}(t)=\sum_{i=1}^{n} \beta_{i} t_{i}$ The sets $\left\{\lambda_{i}\right\},\left\{\alpha_{i}\right\},\left\{\beta_{i}\right\}$ are the set of eigenvalues of $C, A$, and $B$ respectively.

\section{A Generalization of TNCL (GTNCL)}

In this section we will show how a generalization of TNCL can be formulated. In the proof of the TNCL in [2] and [3] we took advantage of the facts that the set

$$
C=\left\{\left(t_{i}\right) \in l_{1}^{+}: \sum t_{i}=1\right\}
$$

is a convex set and the function 


$$
F\left(g_{1}(\mathbf{t}), g_{2}(\mathbf{t}), \cdots g_{m}(\mathbf{t})\right)
$$

is a self-reducing function. A function

$$
F\left(t_{1}, t_{2}, \cdots t_{n}\right)
$$

can be a self-reducing function without being composed of linear combinations of the form $g_{k}(\mathbf{t})=\sum c_{i}^{k} t_{i}$.

Example 3 Consider the function

$$
F\left(t_{1}, t_{2}, \cdots t_{n}\right)=\left(1-\mathrm{e}^{-t_{1}}\right)+\left(1-\mathrm{e}^{-t_{2}}\right)+\left(1-\mathrm{e}^{-t_{3}}\right)+\cdots+\left(1-\mathrm{e}^{-t_{n}}\right) .
$$

This function is self-reducing but is not a composition of linear combinations. A close look at our arguments in [3] shows that the only property used was the fact that the function to be minimized was self-reducing and the set $\sum_{i=1}^{n} t_{i}=1$ was convex. Therefore, we can state the following lemma which is a generalization of TNCL. We state the lemma for the case that the number of variables in finite (a case which occurs in many applied problems) but the arguments used in [3] show that the lemma is also valid in the case where the number of variables is infinite.

Lemma 4 If the function

$$
F\left(t_{1}, t_{2}, \cdots t_{n}\right)
$$

is a positive self-reducing function on the convex set

$$
C=\left\{\left(t_{i}\right) \in l_{1}^{+}: \sum t_{i}=1\right\}
$$

then the minimizing vectors

$$
t=\left(t_{1}, t_{2}, \cdots t_{n}\right)
$$

have at most two nonzero components.

We call the lemma stated above the General Two Nonzero Component Lemma or GTNCL for short.

Remark 5 We can also use TNCL and GTNCL to find the maximum of a positive self-reducing function on (19). To see this please note that if

$$
F\left(t_{1}, t_{2}, \cdots t_{n}\right)
$$

is a positive self-reducing function so is

$$
\frac{1}{F\left(t_{1}, t_{2}, \cdots t_{n}\right)}
$$

and maximum of (20) on (19) is the reciprocal of the minimum of (21) on (19).

A general resource allocation problem is stated as

$$
\begin{gathered}
\text { minimize } f\left(x_{1}, x_{2}, \cdots, x_{n}\right) \\
\text { subject to } \sum_{i=1}^{n} x_{i}=N, x_{i} \geq 0, i=1,2, \cdots, n .
\end{gathered}
$$

which can be converted to

$$
\begin{gathered}
\text { minimize } g\left(t_{1}, t_{2}, \cdots, t_{n}\right) \\
\text { subject to } \sum_{i=1}^{n} t_{i}=1, t_{i} \geq 0, \quad i=1,2, \cdots, n .
\end{gathered}
$$

In the following sections we will use GTNCL to compute a closed form answer for the distribution of the search effort problem.

\section{The Distribution of the Search Effort}

This problem is formulated as 


$$
\begin{gathered}
\text { minimize } \sum_{i=1}^{n}\left(1-\mathrm{e}^{-\alpha x_{i}}\right) p_{i} \\
\text { subject to } \sum_{i=1}^{n} x_{i}=N, x_{i} \geq 0, i=1,2, \cdots, n .
\end{gathered}
$$

where $\alpha$ is a positive number, $p_{i}$ is the probability of an object being at position $i$ and $\left(1-e^{-\alpha x_{i}}\right) p_{i}$ is the conditional probability of detecting the object at position $i$. If we define

$$
u_{i=\frac{x_{i}}{N}}
$$

then the distribution of the search effort problem will be transformed into

$$
\begin{gathered}
\text { minimize } \sum_{i=1}^{n}\left(1-\mathrm{e}^{-\beta u_{i}}\right) p_{i} \\
\text { subject to } \sum_{i=1}^{n} u_{i}=1, x_{i} \geq 0, \quad i=1,2, \cdots, n .
\end{gathered}
$$

Theorem 6 The minimum of

$$
\sum_{i=1}^{n}\left(1-\mathrm{e}^{-\beta x_{i}}\right) p_{i}
$$

subject to

$$
\sum_{i=1}^{n} u_{i}=1, u_{i} \geq 0, \quad i=1,2, \cdots, n
$$

is either

$$
\left(1-\mathrm{e}^{-\beta}\right) p_{i}
$$

for some $1 \leq i \leq n$ or

$$
p_{i}+p_{j}+\mathrm{e}^{-\frac{1}{2} \beta}\left(p_{i} \sqrt{\frac{p_{j}}{p_{i}}}+p_{j} \sqrt{\frac{p_{i}}{p_{j}}}\right)
$$

for a a pair of $i$ and $j, 1 \leq i \leq n$ and $1 \leq j \leq n$.

Proof. Since

$$
\sum_{i=1}^{n}\left(1-\mathrm{e}^{-\beta u_{i}}\right) p_{i}
$$

is a self -reducing function, the GTNCL can be used to find the minimum of this function subject to

$$
\sum_{i=1}^{n} u_{i}=1, u_{i} \geq 0, \quad i=1,2, \cdots, n
$$

Since $\alpha$ is positive so is $\beta$. Suppose $u_{i} \geq 0, \quad i=1,2, \cdots, n$ are components of a minimizing vector on the feasible set (27). By GTNCL either there is an $i, 1 \leq i \leq n$ so that $u_{i}=1$ and $u_{j}=0$ for $j \neq i$ and $1 \leq i \leq n$ or there is a pair of $i$ and $j, 1 \leq i \leq n, 1 \leq j \leq n$ such that $u_{i} \neq 0, u_{j} \neq 0$ and $u_{k}=o$ for $k \neq i, k \neq j, 1 \leq k \leq n$. In the first case the minimum of (26) on (27) is obviously (24). In the second case the minimum of (26) on (27) is the same as the minimum of

$$
\left(1-\mathrm{e}^{-\beta u_{i}}\right) p_{i}+\left(1-\mathrm{e}^{-\beta u_{j}}\right) p_{j}
$$

subject to

$$
u_{i}+u_{j}=1
$$


Expression (28) can be simplified to

$$
p_{i}+p_{j}-p_{i} \mathrm{e}^{-\beta u_{i}}-p_{j} \mathrm{e}^{-\beta u_{j}}
$$

Substituting $u_{j}=1-u_{i}$ in (30) we have

$$
p_{i}+p_{j}-p_{i} \mathrm{e}^{-\beta u_{i}}-p_{j} \mathrm{e}^{-\beta\left(1-u_{i}\right)}
$$

If we differentiate (31) with respect to $u_{i}$ and put the derivative equal to zero we have

$$
p_{j} \beta \mathrm{e}^{\beta\left(u_{i}-1\right)}-p_{i} \beta \mathrm{e}^{-\beta u_{i}}=0
$$

If we solve (32) for $u_{i}$, we obtain

$$
u_{i}=\frac{1}{2 \beta}\left(\beta+\ln p_{i}-\ln p_{j}\right)=\frac{1}{2 \beta}\left(\beta+\ln \frac{p_{i}}{p_{j}}\right)
$$

Substituting $u_{i}$ from (33) in (29) gives us

$$
u_{j}=\frac{1}{2 \beta}\left(\beta+\ln \frac{p_{j}}{p_{i}}\right)
$$

If we substitute (33) and (34) in (30) we have

$$
p_{i}+p_{j}+p_{i} \mathrm{e}^{-\frac{1}{2}\left(\beta+\ln \frac{p_{i}}{p_{j}}\right)}+p_{j} \mathrm{e}^{-\frac{1}{2}\left(\beta+\ln \frac{p_{j}}{p_{i}}\right)} .
$$

The last expression is equivalent to

$$
p_{i}+p_{j}+\mathrm{e}^{-\frac{1}{2} \beta}\left(p_{i} \sqrt{\frac{p_{i}}{p_{j}}}+p_{j} \sqrt{\frac{p_{j}}{p_{i}}}\right) .
$$

Please note that the derivative of the function

$$
p_{j} \beta \mathrm{e}^{\beta\left(u_{i}-1\right)}-p_{i} \beta \mathrm{e}^{-\beta u_{i}}
$$

with respect to $u_{i}$ is

$$
\beta^{2} p_{j} \mathrm{e}^{\beta\left(u_{i}-1\right)}+\beta^{2} p_{i} \mathrm{e}^{-\beta u_{i}}
$$

which is positive for $u_{i}, 0 \leq u_{i} \leq 1$. Therefore, by the second derivative test (36) is a minimum value not a maximum value for the objective function in the resource allocation problem.

Although the GTNCL states two components $u_{i}$ and $u_{j}$ are nonzero but in general we do not know exactly which pair of $p_{i}$ and $p_{j}$ expresses (36). When applying TNCL to problems of matrix optimization, the author was able to determine exactly which component or which pair of components of the optimizing vectors are nonzero (see [5]) under certain conditions. The same can be done here.

Theorem 7 Suppose the probabilities $p_{i}, 1 \leq i \leq n$ are ordered such that

$$
p_{1} \leq p_{2} \leq p_{3} \cdots \leq p_{n}
$$

Then the minimum of

$$
\sum_{i=1}^{n}\left(1-\mathrm{e}^{-\beta u_{i}}\right) p_{i}
$$

subject to

$$
\sum_{i=1}^{n} u_{i}=1, u_{i} \geq 0, \quad i=1,2, \cdots, n
$$

is

$$
\left(1-\mathrm{e}^{-\beta}\right) p_{i}
$$


Proof. Assume

$$
p_{1} \leq p_{2} \leq p_{3} \cdots \leq p_{n}
$$

Since $\beta>0$ (39) implies that

$$
\min \left\{\left(1-\mathrm{e}^{-\beta}\right) p_{i}\right\}_{i=1}^{n}=\left(1-\mathrm{e}^{-\beta}\right) p_{1}
$$

Furthermore, in (25) assume $i<j$. Let $x=\frac{p_{j}}{p_{i}}$ and $k=\mathrm{e}^{-\frac{1}{2} \beta}$. Obviously $x \geq 1$. Now (25) can be written as

$$
p_{i}\left[1+x+k\left(\frac{\sqrt{x}}{x}+x \sqrt{x}\right)\right]
$$

Note that $k>0$. If we define

$$
f(x)=1+x+k\left(\frac{\sqrt{x}}{x}+x \sqrt{x}\right)
$$

then

$$
\frac{\mathrm{d} f(x)}{\mathrm{d} x}=\frac{1}{2 x^{\frac{3}{2}}}\left(3 k x^{2}-k+2 x^{\frac{3}{2}}\right)
$$

Since $x \geq 1$ then

$$
3 k x^{2}-k+2 x^{\frac{3}{2}}>3 k-k+2=2 k+2>0
$$

This shows that $f(x)$ is an increasing function on $[1, \infty)$. Hence on the finite set

$$
\left\{\frac{p_{i+1}}{p_{i}}, \frac{p_{i+2}}{p_{i}}, \cdots \frac{p_{n-1}}{p_{i}}\right\}
$$

the function

$$
f(x)=1+x+k\left(\frac{\sqrt{x}}{x}+x \sqrt{x}\right)
$$

has its minimum at $\frac{p_{i+1}}{p_{i}}$. Therefore, if two components $u_{i}$ and $u_{j}$ are nonzero, we must have $j=i+1$ and in this case the minimum of the objective function is

$$
p_{i}+p_{i+1}+\mathrm{e}^{-\frac{1}{2} \beta}\left(p_{i} \sqrt{\frac{p_{i+1}}{p_{i}}}+p_{j} \sqrt{\frac{p_{i}}{p_{i+1}}}\right)
$$

for some $i, 1 \leq i \leq n$. Next notice that

$$
\left(1-\mathrm{e}^{-\beta}\right) p_{1}<p_{1}<p_{i}+p_{i+1}+\mathrm{e}^{-\frac{1}{2} \beta}\left(p_{i} \sqrt{\frac{p_{i+1}}{p_{i}}}+p_{j} \sqrt{\frac{p_{i}}{p_{i+1}}}\right)
$$

for $1 \leq i \leq n$. Hence the minimum of the objective function is

$$
\left(1-\mathrm{e}^{-\beta}\right) p_{1} \text {. }
$$

Since both TNCL and GTNCL are valid for infinite number of variables, these techniques can be used to solve resource allocatoin problems involving an infinite number of variables as well. For example, in the distribution of the search effort problem we can assume the search is for an object on the plane that can be potentially 
detected at an infinite set of locations (such as points with integer $x$ and $y$ coordinates).

\section{Optimal Portfolio Selection}

There are other resource allocation problems that we are able to tackle with GTNCL, One of these problems is the problem of optimal portfolio selection. One model for this general problem is formulated as finding the maximum of

$$
\frac{\left(\sum_{i=1}^{n} R_{i} x_{i}\right)}{\left(\sum \sigma_{i j} x_{i} x_{j}\right)^{\frac{1}{2}}}
$$

$$
\text { subject to } \sum_{i=1}^{n} x_{i}=1, x_{i} \geq 0, i=1,2, \cdots, n \text {. }
$$

(see [6]). Here $R_{i}$ is expected return on security $i$ and $\sigma_{i j}$ is the covariance between securities $i$ and $j$. If the correlation coefficients between $i$ and $j$ are constant $\rho$, with $0<\rho<1$, then $\sigma_{i i}=\sigma_{i}^{2}$, $\sigma_{i j}=\rho \sigma_{i} \sigma_{j}$ and from Karush-Kuhn-Tucker conditions the problem is reduced to

$$
\begin{gathered}
\text { maximize } \sum_{i=1}^{n}\left\{\left(R_{i}-\frac{1}{2} \sigma\right) x_{i}-\frac{(1-\rho)}{2} \sigma_{i}^{2} x_{i}^{2}\right\} \\
\text { subject to } \sum_{i=1}^{n} x_{i}=1, x_{i} \geq 0, i=1,2, \cdots, n .
\end{gathered}
$$
it.

Notice that (44) is a self-reducing function and one can again apply the GTNCL to find a maximum value for

The problems of distribution of search effort and optimal portfolio selection are both examples of separable resource allocation problems. A separable resource allocation problem is a problem where we want to minimize or maximize

$$
\begin{gathered}
\sum_{i=1}^{n} f_{i}\left(x_{i}\right) \\
\text { subject to } \sum_{i=1}^{n} x_{i}=N, x_{i} \geq 0, i=1,2, \cdots, n .
\end{gathered}
$$

where each $f_{i}$ is continuously differentiable over an interval including $[0, N]$. The GTNCL can be applied to such a problem if $f_{i}(0)=0$, for each $i, 1 \leq i \leq n$. This condition is not satisfied for a number of resource allocation problems including optimal sample allocation in stratified sampling, and production planning (see [5]).

Remark 8 In a broader sense, each Kantorovich-type matrix optimization problem such as the one in Example 2 can be regarded as a resource allocation problem where our resource is just the set of pure numbers on the interval [0,1]. For instance in Example 2 the problem is reduced to finding minimum of

$$
\begin{gathered}
\frac{\left(\sum_{i=1}^{n} \lambda_{i} t_{i}\right)^{2}}{\left(\sum_{i=1}^{n} \alpha_{i} t_{i}\right)\left(\sum_{i=1}^{n} \beta_{i} t_{i}\right)} \\
\text { Subject to } \sum_{i=1}^{n} t_{i}=1
\end{gathered}
$$

Each $t_{i}=\left\|z_{i}\right\|^{2}$, where each $z_{i}$ is a component of a minimizing vector $f$ of norm 1 for the operator optimization problem (15). Indeed nonzero components of a minimizing vector $f$ are important in applica- 
tions. Historically, the optimization problem (15) was first discussed by R. Cameron and B. Kouvartakis in an effort to minimize the norm of output feedback controllers used in pole placement (see [7]).

Remark 9. The author is not aware of any other theorem that provides closed form answers for resource allocation problems. The results we obtain might be of interest for instance in signal analysis where one needs to minimize the resource spent finding a signal that is probable to detected at a certain location. Computer models are used for solving such problems and it is interesting to investigate how consistent the results of computer models are with our results here. Also, many theories in portfolio selection suggest that diversification maximizes the profit. At the first glance this might sound inconsistent with the results one might obtain using the two nonzero theorem. However, we have to remember that the over theory also ensures diversification increases profit. If the profit is maximized for one or two securities, then the more the number of securities, the more pairs of securities we have.

\section{References}

[1] Gustafson, K. and Rao, D. (1997) Numerical Range. Springer, New York. http://dx.doi.org/10.1007/978-1-4613-8498-4

[2] Gustafson, K. and Seddighin, M. (1989) Antieigenvalue Bounds. Journal of Mathematical Analysis and Applications, 143, 327-340. http://dx.doi.org/10.1016/0022-247X(89)90044-9

[3] Seddighin, M. (2002) Antieigenvalues and Total Antieigenvalues of Normal Operators. Journal of Mathematical Analysis and Applications, 274, 239-254. http://dx.doi.org/10.1016/S0022-247X(02)00295-0

[4] Seddighin, M. (2009) Antieigenvalue Techniques in Statistics. Linear Algebra and Its Applications, 430, $2566-2580$. http://dx.doi.org/10.1016/j.laa.2008.05.007

[5] Seddighin, M. and Gustafson, K. (2005) On the Eigenvalues which Express Antieigenvalues. International Journal of Mathematics and Mathematical Sciences, 2005, 1543-1554. http://dx.doi.org/10.1155/IJMMS.2005.1543

[6] Ibaraki, T. and Katoh, N. (1988) Resource Allocation Problems. The MIT Press, Cambridge.

[7] Cameron, R. and Kouvartakis, B. (1980) Minimizing the Norm of Output Feedback Controllers Used in Pole Placement: A Dyadic Approach. International Journal of Control, 32, 759-770. http://dx.doi.org/10.1080/00207178008922889 
Scientific Research Publishing (SCIRP) is one of the largest Open Access journal publishers. It is currently publishing more than 200 open access, online, peer-reviewed journals covering a wide range of academic disciplines. SCIRP serves the worldwide academic communities and contributes to the progress and application of science with its publication.

Other selected journals from SCIRP are listed as below. Submit your manuscript to us via either submit@scirp.org or Online Submission Portal.
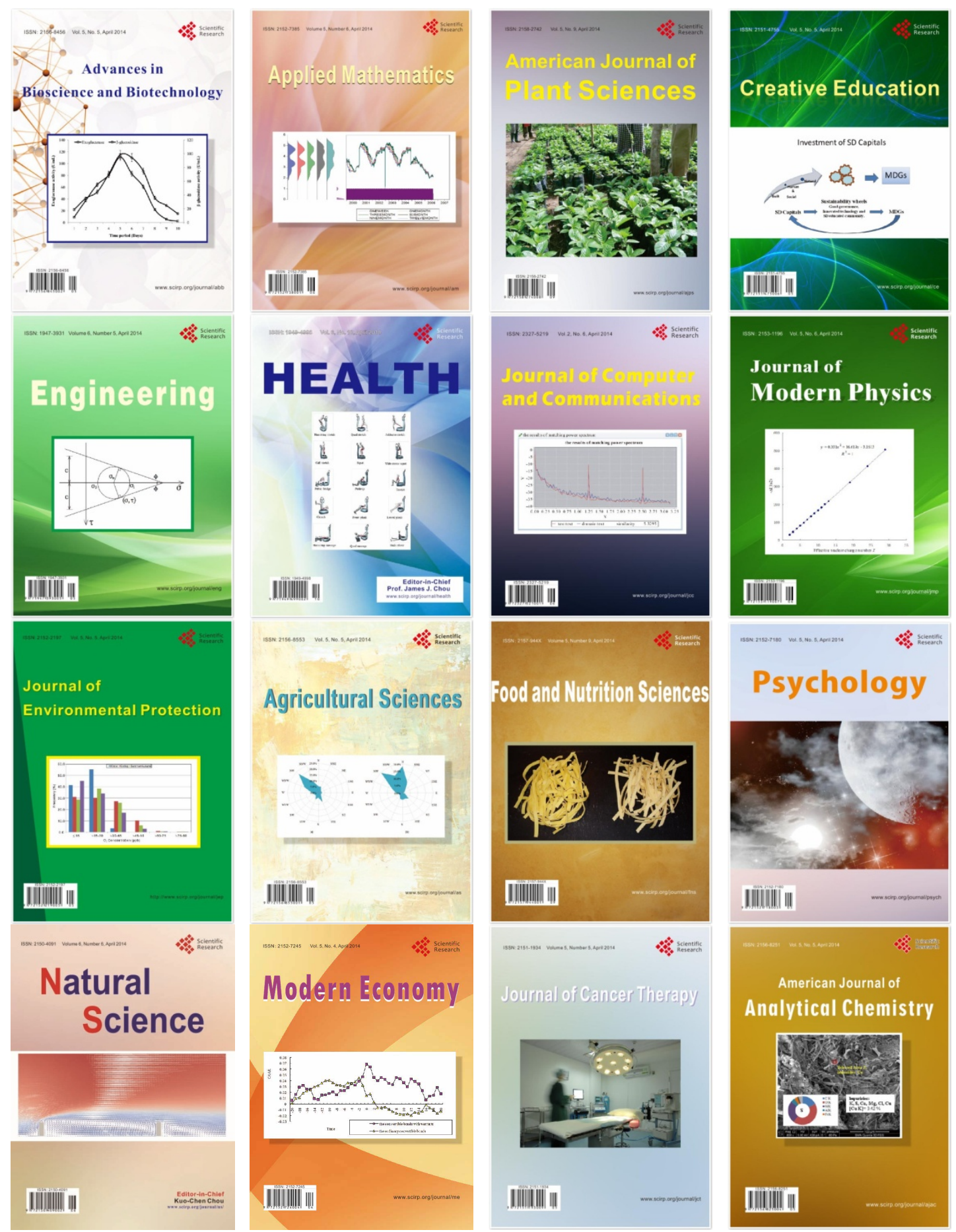